\title{
THERMOPHILE ENDOSPORES HAVE RESPONSIVE EXOSPORIUM FOR ATTACHMENT ${ }^{*}$
}

B. Panessa-Warren ${ }^{\dagger}$, George T. Tortora ${ }^{\ddagger}$, J. Warren ${ }^{*}$, and R. Sabatini ${ }^{\alpha}$

${ }^{\dagger}$ Graduate Division, School of Health Technology \& Management

SUNY, Stony Brook, NY 11794-8204

${ }^{\ddagger}$ Clinical Microbiology Lab., University Hospital and

Dept. of Clinical Lab. Sciences,

School of Health Technology \& Management

SUNY, Stony Brook, NY 11794-8204

"Instrumentation Division

Brookhaven National Laboratory

Upton, NY 11973-5000

${ }^{\propto}$ Materials Science

Brookhaven National Laboratory

Upton, NY 11973-5000

'August, 1999

*Work supported in part by the U.S. Department of Energy Contract No. DE-AC02-98CH10886. 


\section{THERMOPHILE ENDOSPORES HAVE RESPONSIVE EXOSPORIUM FOR ATTACHMENT}

B.Panessa-Warren*, George T.Tortora**, J.Warren+ and R.Sabatinit-t

*Graduate Division, School of Health Technology \& Management, SUNY, Stony Brook,N.Y.11794-8204

**Clinical Microbiology Lab. University Hospital and Dept. of Clinical Lab Sciences, School of Health Technology \& Management, SUNY,Stony Brook,N.Y.11794-8205

+Instrumentation Division, Brookhaven National Lab, Upton, N.Y.11973

++Materials Science, Brookhaven National Lab, Upton, N.Y. 11973

Recently studies examining the colonization of Clostridial pathogens on agar and human tissue culture cells, ${ }^{1,2.3}$ demonstrated that ( $\underline{C}$. sporogenes ATCC 3584, C. difficile ATCC 43594 [patient isolate], . difficile ATCC 9689 [non-clinical], C. clostridioforme [patient isolate]) bacterial spores (endospores) of the genus Clostridia have an outer membrane that becomes responsive at activation and exhibits extensions of the exosporial membrane that facilitate and maintain spore attachment to a nutritive substrate during germination and initial outgrowth of the newly developed bacterial cell. Therefore this attachment phenomenon plays an important role in insuring bacterial colonization of a surface and the initial stages of the infective process. To see if other non-clinical members of this genus also have this ability to attach to a substrate or food-source during spore germination, and how this attachment process in environmental thermophiles compares to the clinical paradigm (in relation to time sequence, exosporial membrane structure, type of attachment structures, composition of the membrane etc...), sediment samples were collected in sterile transport containers at 4 geothermal sites at Yellowstone National Park in Wyoming. Because spore forming bacteria will produce spores when conditions are unfavorable for growth, the samples were sealed and stored at $4^{\circ} \mathrm{C}$. After 8 months the samples were screened for the presence of spores by light microscope examination using malachite green $/ \mathrm{safranin}^{4}$, and traditional endospores were identified in significant quantities from the Terrace Spring site $\left(a 46^{\circ} \mathrm{C}\right.$ lake with bacterial mats and a rapidly moving run-off channel leading to a traditional hot spring). The highest spore population was found in the top sediment and benthic water of the run-off channel, $\mathrm{pH} \mathrm{8.1.}$

Thermophile spores were grown on pre-reduced Trypticase soy agar with $0.5 \%$ glucose in anaerobic bags at $56^{\circ} \mathrm{C}$ and $37^{\circ} \mathrm{C}$. Considerable growth was noted on plates within 4 hours of incubation, which is consistent with the rapid rate of growth noted for thermophiles. Plates were anaerobically examined at 4, 18, 24, 48 and $72 \mathrm{hrs}$. To insure maximum spore production samples for light and electron microscopy were taken at 14 days post inoculation and either prepared (Gram stain, Malachite green/safranin) as smears for light microscopy; or fixed in $3 \%$ glutaraldehyde in $0.1 \mathrm{M}$ cacodylate buffer with $0.1 \% \mathrm{CaCl}_{2}$, washed in buffer, dehydrated in acetone, dried by the critical point method and rotary coated with $6 \mathrm{~nm}$ AuPd. SEM samples were examined at 20 or $17 \mathrm{KV}$ accelerating voltage on an AMRay SEM $1000 \mathrm{~A}$ with a $\mathrm{LaB}_{6}$ gun, or a JSM 6400 tungsten thermionic gun.

At both temperatures, bacterial spores were observed that had the traditional histochemical green spore protoplast and red exosporial membrane. Exosporia were observed attached to each other, debris (fragments of dead vegetative cells, empty exosporia and agar), and attached to the light microscope slide. By SEM myriads of spores were found. Samples from $56^{\circ} \mathrm{C}$ incubated plates showed fields of spores and vegetative cells enmeshed in a fibrous network. Individual spores showed numerous delicate attachments, but the exosporial membrane showed only modest surface extensions. The same samples grown at $37^{\circ} \mathrm{C}$ formed a bacterial mat with many vegetative cells and spores embedded in a dense layer of fibrous and amorphous material. Both vegetative cells and spores lay in small excavated lacunae in this layer of material, similar to the way in which $\underline{C}$. difficile and $\underline{C}$. sporogenes are surrounded by a clear zone when they are grown on agar. The exosporial membrane of the $37^{\circ} \mathrm{C}$ grown samples revealed numerous exosporial surface projections, identical to those of $\underline{C}$. sporogenes and $\underline{C}$. clostridioforme. 
We have found a survival mechanism (attachment of bacterial endospores to nutritive surfaces to facilitate colonization and survival) in mesophiles (human pathogens) that we can now demonstrate in thermophiles... indicating that this "attachment phenomenon" may be common to all Clostridia and perhaps even to other bacterial spores. The thermophile exosporial membrane seems to function similarly to that of the mesophilic endospores, but there is a much more rapid attachment cycle taking minutes, rather than 1.5 to 4 hrs for attachment. This data poses very provocative questions for further study. Does the exosporial membrane respond with extensive surface appendages at one temperature and not at the higher temperature? Since the upper limit for thermophile growth is determined by thermolability of biomolecules, organellar membranes, and enzyme systems ${ }^{5}$, understanding how the exosporial membrane of the thermophile differs from the mesophile in morphology, function and composition at specific temperatures will provide new information on biomembrane mutability in regard to environmental diversity.

\section{References}

1.B.Panessa-Warren, G. Tortora \&J.Warren,SCANNING, 16 (1994) 227.

2.B.Panessa-Warren, G.Tortora \&J.Warren,Tissue \& Cell,29 (1997)441.

3.G.Tortora,B.Panessa-Warren \&J.Warren, Proc.Amer.Soc. Microbiol. (1998)234.

4.X.Schaeffer \& Fulton, Science 77(1933) 194.

5.D.Schwartzman, The Keys to Molecular Evolution and the Origin of Life, ed. J.Wiegel \& M.Adams (1998)(In Press).

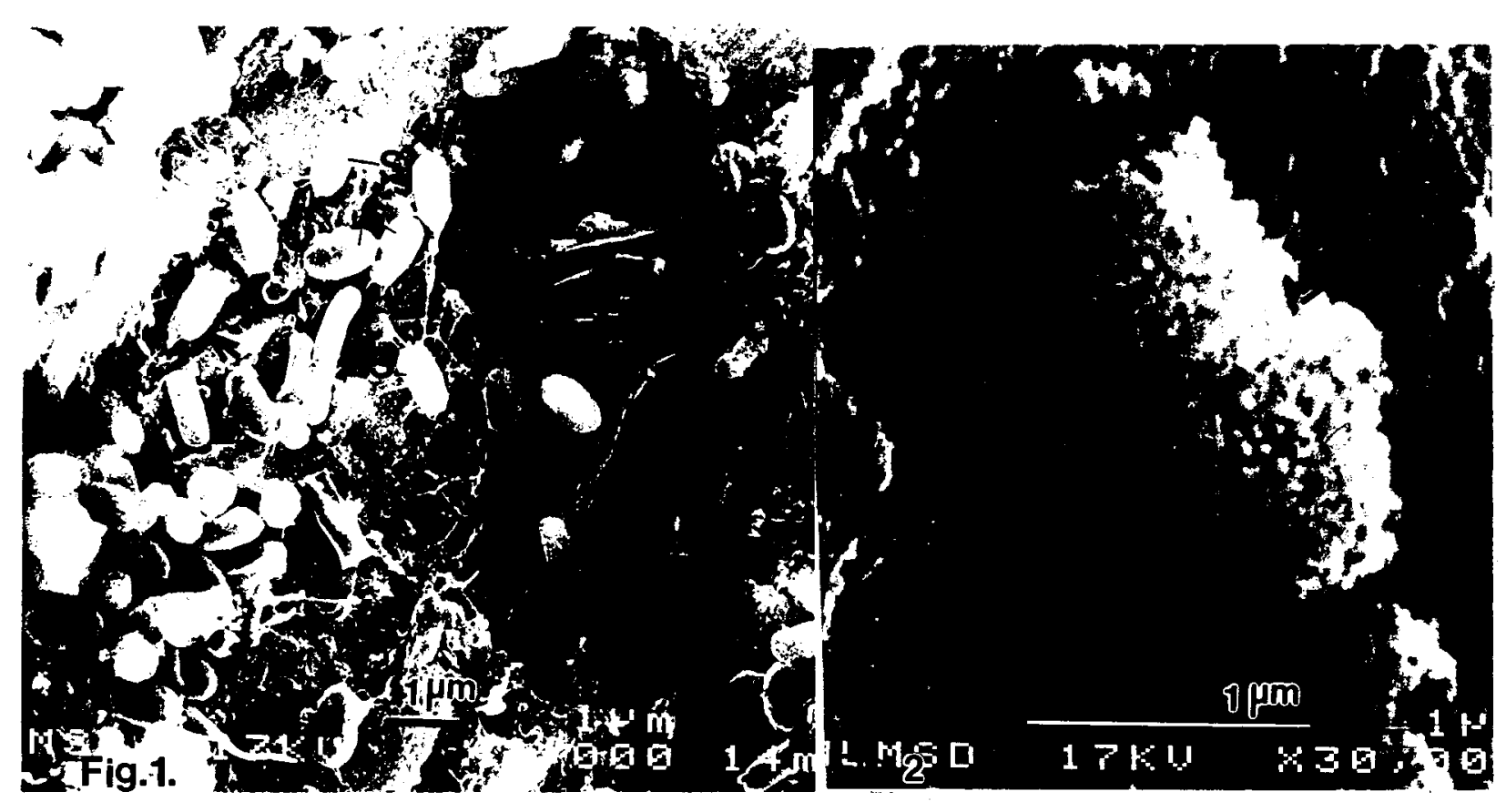

FIG.1. Thermophile endospores grown at $56^{\circ} \mathrm{C}$. Spore (S) attachment structures (arrows).

FIG.2. Thermophile spore (S) with activated exosporium (arrows) $37^{\circ}$. 